CRYSTALLOGRAPHIC COMMUNICATIONS

ISSN 2056-9890

Received 29 November 2017

Accepted 22 January 2018

Edited by W. T. A. Harrison, University of Aberdeen, Scotland

Keywords: crystal structure; osmium(II) complex; tropolonato; cymene ligand.

CCDC reference: 1818437

Supporting information: this article has supporting information at journals.iucr.org/e

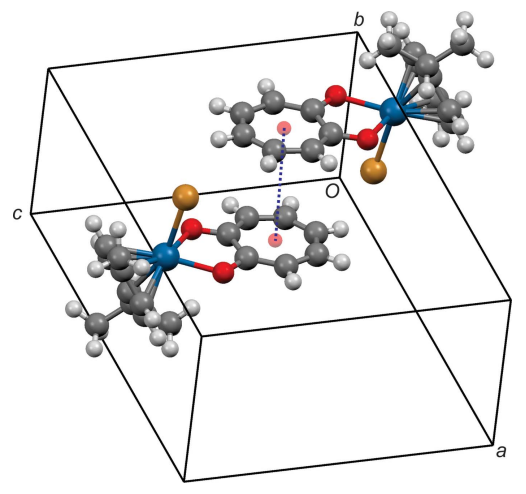

OPEN $\odot$ ACCESS

\section{Crystal structure of bromido $\left(\eta^{6}-1\right.$-isopropyl-4- methylbenzene)(7-oxocyclohepta-1,3,5-trien-1- olato- $\left.\kappa^{2} O, O^{\prime}\right)$ osmium}

\author{
Hadley S. Clayton, ${ }^{a *}$ Kgaugelo C. Tapala ${ }^{a}$ and Andreas Lemmerer ${ }^{b}$
}

${ }^{a}$ Chemistry Department, University of South Africa, Pretoria, 0003, South Africa, and ${ }^{\mathbf{b}}$ Molecular Sciences Institute, School of Chemistry, University of the Witwatersrand, Private Bag, PO WITS, 2050, Johannesburg, South Africa.

*Correspondence e-mail: clayths@unisa.ac.za

In the title compound, $\left[\mathrm{OsBr}\left(\mathrm{C}_{10} \mathrm{H}_{14}\right)\left(\mathrm{C}_{7} \mathrm{H}_{5} \mathrm{O}_{2}\right)\right]$, the central $\mathrm{Os}^{\mathrm{II}}$ ion is ligated by a hexahaptic $\eta^{6} p$-cymene ring, a $\mathrm{Br}^{-}$ligand and two $\mathrm{O}$ atoms of a chelating tropolonate group. The $p$-cymene ligand presents more than one conformation, giving rise to a discrete disorder, which was modelled with two different orientations with occupancy values of $0.561(15)$ and 0.439 (15). The crystal packing features $\mathrm{C}-\mathrm{H} \cdots \mathrm{O}$ and $\mathrm{C}-\mathrm{H} \cdots \mathrm{Br}$ hydrogen bonding. Aromatic $\pi-\pi$ stacking interactions are also observed between adjacent non-benzenoid aromatic tropolone rings.

\section{Chemical context}

The chemistry of half-sandwich organometallic $\mathrm{Os}^{\mathrm{II}}$-arene complexes with O-donor ligands has drawn considerable interest because of their potential application as anticancer agents (Zhang \& Sadler, 2017). In particular, several complexes of this type with $O, O$ - and $N, O$-chelating ligands have been investigated (Hanif et al., 2010; van Rijt et al., 2009). While the complexes with $\mathrm{N}, \mathrm{O}$-ligands have shown in vitro anticancer activity comparable to Cisplatin, the benchmark anticancer metallopharmaceutical, complexes with $O, O$ ligands exhibit low activity. This has been attributed to the poor stability of these complexes in aqueous solution and the formation of inactive hydroxy-bridged dimers (Hanif et al., 2014). The mechanism of the cytotoxic action of the OsI ${ }^{\mathrm{II}}$ arene complexes is generally thought to involve hydrolysis of the Os $-X$ bond (where $X=$ a halide ligand) to generate an active $\mathrm{Os}-\mathrm{OH}_{2}$ species, which binds to biomolecules leading to cellular dysfunction and consequently triggering apoptosis. While the anticancer activity of the $\mathrm{Os}^{\mathrm{II}}$-arene complexes has often been compared to that of their $\mathrm{Ru}$ analogues, no definitive structure-activity relationship has yet been elucidated. In addition, the Os ${ }^{\mathrm{II}}$-arene complexes appear to have an altered pharmacological profile in comparison with the ruthenium complexes (Bruijnincx \& Sadler, 2009). As part of our studies in this area, single-crystal X-ray diffraction was used to determine the structure of the title compound, (I).

\section{Structural commentary}

The molecular structure of (I) is shown in Fig. 1 and selected geometrical data are presented in Table 1. The complex adopts a 'three-legged piano-stool' geometry, where the $\eta^{6}$-coord- 
inated arene ring is present as the seat, and the two $\mathrm{O}$ atoms of the tropolonate ligand along with the bromido ligand as the three legs of the stool.

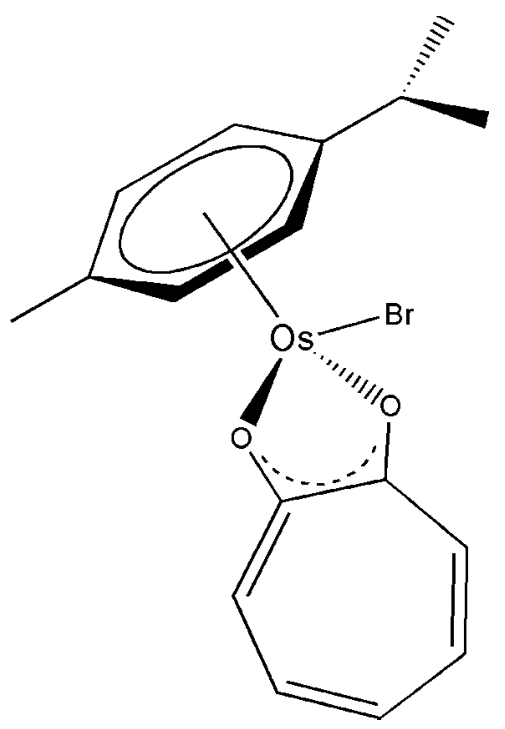

The tropolonato anion is chelated to the metal centre, forming a five-membered $\mathrm{OsO}_{2} \mathrm{C}_{2}$ ring, which is almost planar, with the tight bite angle $\left[76.3(2)^{\circ}\right]$ of the tropolonate chelate resulting in a distorted pseudo-octahedral coordination sphere. The rigid tropolonate ligand backbone is made up of an almost planar seven-membered ring consisting of conjugated $\mathrm{sp}^{2}$ carbon atoms. The Os-O bond lengths [2.071 (6)

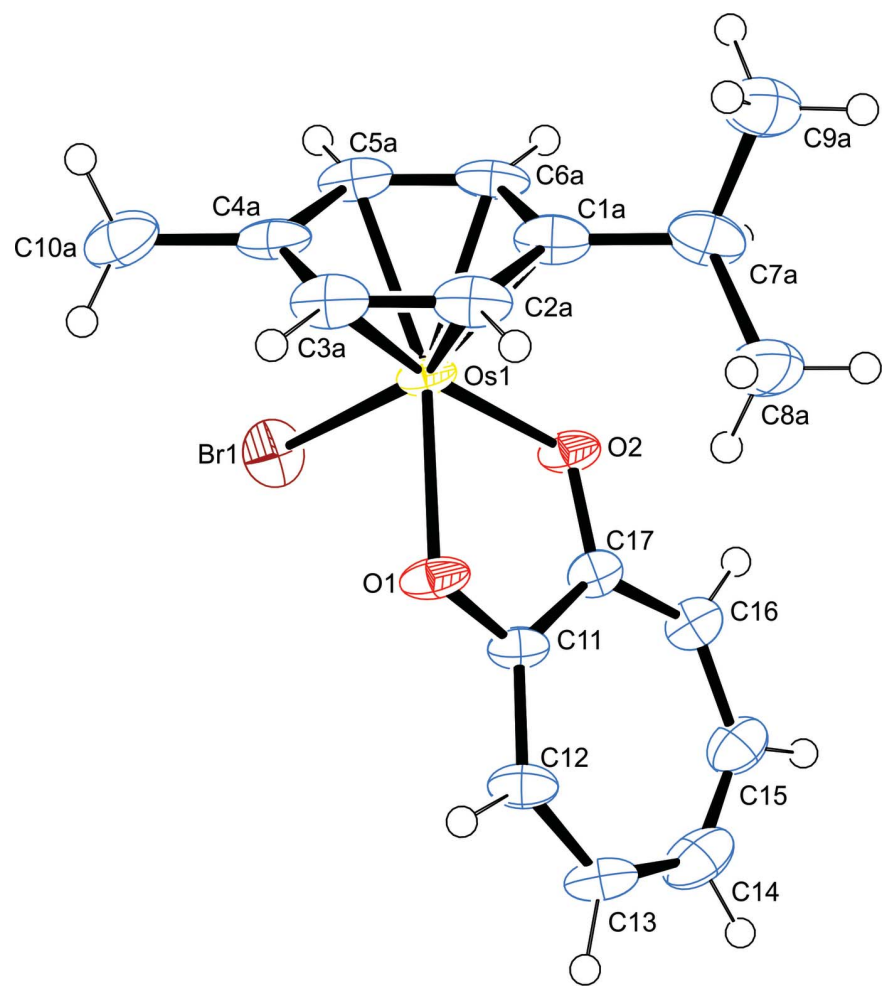

Figure 1

The molecular structure of (I) showing 50\% displacement ellipsoids. Only one orientation of the disordered benzene ring is shown.
Table 1

Selected bond lengths $(\AA)$.

\begin{tabular}{llll}
\hline Os1-C1A & $2.090(12)$ & Os1-C3B & $2.167(19)$ \\
Os1-C2A & $2.125(14)$ & Os1-C4B & $2.192(16)$ \\
Os1-C3A & $2.158(14)$ & Os1-C5B & $2.21(2)$ \\
Os1-C4A & $2.157(12)$ & Os1-C6B & $2.21(2)$ \\
Os1-C5A & $2.123(18)$ & Os1-O1 & $2.088(6)$ \\
Os1-C6A & $2.089(18)$ & Os1-O2 & $2.071(6)$ \\
Os1-C1B & $2.187(13)$ & Os1-Br1 & $2.5472(12)$ \\
Os1-C2B & $2.164(17)$ & & \\
\hline
\end{tabular}

and 2.088 (6) $\AA$ ] are similar to those of the related ruthenium compound ( $c$ a $2.1 \AA$ ) published previously (Dwivedi et al., 2016). The isobidentate nature of the $\mathrm{OsO}_{2} \mathrm{C}_{2}$ moiety is evidence of delocalization of the $\mathrm{C}=\mathrm{O}$ bonds of the tropolone ligand upon coordination $[\mathrm{C} 11-\mathrm{O} 1=1.303(11), \mathrm{C} 17-\mathrm{O} 2=$ $1.299(11) \AA]$. The aromatic ring of the $p$-cymene ligand appears almost planar, with the displacement of the arene ring centroid from the Os ${ }^{\mathrm{II}}$ center [1.676 ̊] being comparable with other similar complexes (Peacock et al., 2007; Kandioller et al. 2013).

\section{Supramolecular features}

In the crystal, the coordinated $\mathrm{O}$ atoms of the tropolonate ligand accept weak $\mathrm{C}-\mathrm{H}$. . O interactions (Table 2) from the $p$-cymene ring in the range $2.40-2.72 \AA$, which contribute to the crystal packing. In addition, the bromide ion acts as a hydrogen-bond acceptor, forming $\mathrm{C}-\mathrm{H} \cdots \mathrm{Br}$ hydrogen bonds with a $\mathrm{C}-\mathrm{H}$ group from the arene ring of an adjacent molecule. There is also a $\pi-\pi$ stacking interaction between the tropolone ligands with the plane-to-plane distances of the stacked aromatic ring moieties at $3.895 \AA$ (Fig. 2).

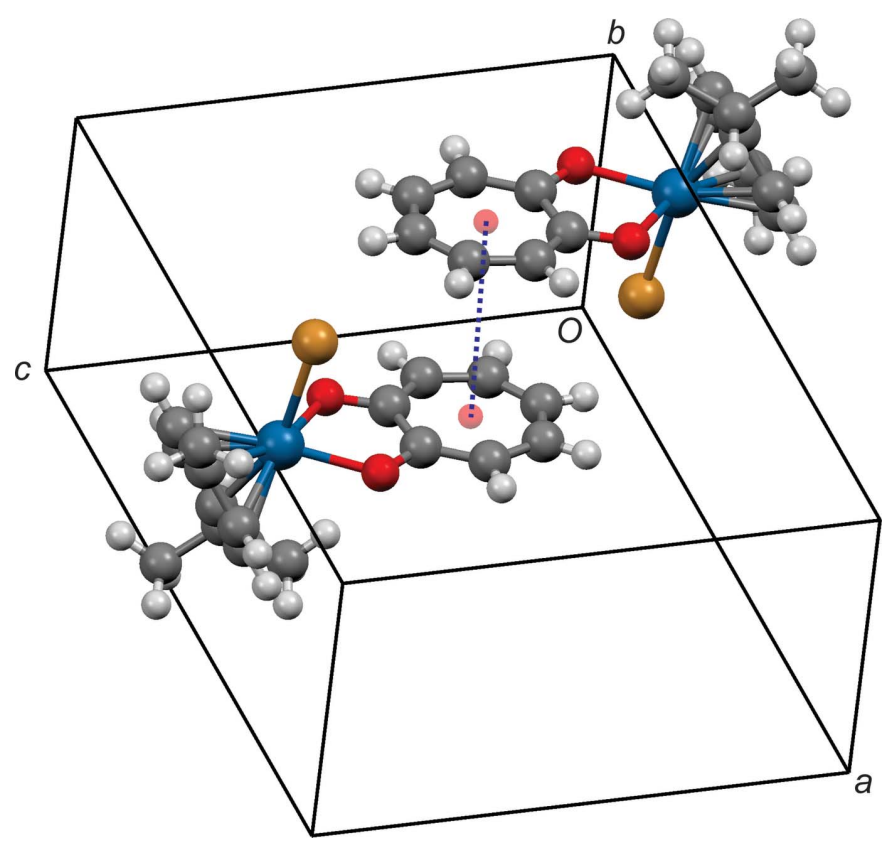

Figure 2

Detail of the packing of (I) showing aromatic $\pi-\pi$ stacking between the seven-membered rings as a blue dashed line. 
Table 2

Hydrogen-bond geometry $\left(\AA,^{\circ}\right)$.

\begin{tabular}{lllll}
\hline$D-\mathrm{H} \cdots A$ & $D-\mathrm{H}$ & $\mathrm{H} \cdots A$ & $D \cdots A$ & $D-\mathrm{H} \cdots A$ \\
\hline $\mathrm{C} 2 A-\mathrm{H} 2 A \cdots \mathrm{O} 1^{\mathrm{i}}$ & 0.95 & 2.40 & $3.24(2)$ & 148 \\
$\mathrm{C} 3 B-\mathrm{H} 3 B \cdots \mathrm{O} 1^{\mathrm{i}}$ & 0.95 & 2.75 & $3.38(2)$ & 124 \\
$\mathrm{C} 5 B-\mathrm{H} 5 B \cdots \mathrm{O} 2^{\mathrm{ii}}$ & 0.95 & 2.50 & $3.25(2)$ & 136 \\
$\mathrm{C} 6 A-\mathrm{H} 6 A \cdots \mathrm{O} 2^{\mathrm{ii}}$ & 0.95 & 2.71 & $3.39(2)$ & 124 \\
$\mathrm{C} 5 A-\mathrm{H} 5 A \cdots \mathrm{O} 2^{\mathrm{ii}}$ & 0.95 & 2.77 & $3.39(2)$ & 124 \\
\hline
\end{tabular}

Symmetry codes: (i) $-x,-y+2,-z$; (ii) $-x+1,-y+2,-z$.

\section{Database survey}

A search of the Cambridge Structural Database (Version 5.38, update February 2017; Groom et al., 2016) for related structures revealed that the isostructural ruthenium complex, $\left[\left(\eta^{6}-\right.\right.$ p-cymene)Ru(trop)Cl] (OTIMOV; Dwivedi et al., 2016), and similar osmium complexes (QEYXIC; Peacock et al., 2007 and BENYUQ; Kandioller et al., 2013) have been reported.

\section{Synthesis and crystallization}

All synthetic procedures were carried out using standard Schlenk techniques under an atmosphere of argon. The osmium dimer $\left[\mathrm{Os}\left(\eta^{6}-p \text {-cymene }\right) \mathrm{Br}_{2}\right]_{2}(1.037 \mathrm{~g}, 1.07 \mathrm{mmol})$ and sodium tropolonate $(0.448 \mathrm{~g}, 3.11 \mathrm{mmol})$ were suspended in methanol $(100 \mathrm{ml})$. The suspension was stirred at room

Table 3

Experimental details.

\begin{tabular}{|c|c|}
\hline \multicolumn{2}{|l|}{ Crystal data } \\
\hline Chemical formula & $\mathrm{C}_{17} \mathrm{H}_{19} \mathrm{BrO}_{2} \mathrm{Os}$ \\
\hline$M_{\mathrm{r}}$ & 525.43 \\
\hline Crystal system, space group & Monoclinic, $P 2_{1} / c$ \\
\hline Temperature $(\mathrm{K})$ & 173 \\
\hline$a, b, c(\AA)$ & $\begin{array}{l}11.1574(5), 14.6104(7), \\
\quad 10.8342(5)\end{array}$ \\
\hline$\beta\left({ }^{\circ}\right)$ & $110.454(2)$ \\
\hline$V\left(\AA^{3}\right)$ & $1654.78(13)$ \\
\hline$Z$ & 4 \\
\hline Radiation type & Mo $K \alpha$ \\
\hline$\mu\left(\mathrm{mm}^{-1}\right)$ & 10.12 \\
\hline Crystal size $(\mathrm{mm})$ & $0.12 \times 0.10 \times 0.05$ \\
\hline \multicolumn{2}{|l|}{ Data collection } \\
\hline Diffractometer & $\begin{array}{l}\text { Bruker D8 Venture Photon CCD } \\
\text { area detector }\end{array}$ \\
\hline Absorption correction & $\begin{array}{l}\text { Integration (XPREP; Bruker, } \\
\text { 2016) }\end{array}$ \\
\hline$T_{\min }, T_{\max }$ & $0.538,0.714$ \\
\hline $\begin{array}{l}\text { No. of measured, independent and } \\
\text { observed }[I>2 \sigma(I)] \text { reflections }\end{array}$ & $58616,3990,3614$ \\
\hline$R_{\text {int }}$ & 0.079 \\
\hline$(\sin \theta / \lambda)_{\max }\left(\AA^{-1}\right)$ & 0.660 \\
\hline \multicolumn{2}{|l|}{ Refinement } \\
\hline$R\left[F^{2}>2 \sigma\left(F^{2}\right)\right], w R\left(F^{2}\right), S$ & $0.053,0.118,1.15$ \\
\hline No. of reflections & 3990 \\
\hline No. of parameters & 259 \\
\hline No. of restraints & 384 \\
\hline H-atom treatment & H-atom parameters constrained \\
\hline$\Delta \rho_{\max }, \Delta \rho_{\min }\left(\mathrm{e} \AA^{-3}\right)$ & $5.63,-2.07$ \\
\hline
\end{tabular}

Computer programs: APEX3, SAINT-Plus and XPREP (Bruker, 2016), SHELXS97 (Sheldrick, 2008) and SHELXL2014/7 (Sheldrick, 2015), ORTEP for Windows and Win $G X$ publication routines (Farrugia, 2012). temperature overnight to give a dark-brown solution. The solution was filtered and the solvent was removed in vacuo. The residue was extracted with $\mathrm{CH}_{2} \mathrm{Cl}_{2}(80 \mathrm{ml})$. The solvent was removed under reduced pressure to give the title compound as a red-brown solid. Yield $72 \% \quad(0.807 \mathrm{~g}$, $1.54 \mathrm{mmol}$ ). Red blocks of (I) were obtained by slow evaporation from a concentrated dichloromethane solution at room temperature over several days.

\section{Refinement}

Crystal data, data collection and structure refinement details are summarized in Table 3. The $\mathrm{C} 1-\mathrm{C} 10$ atoms of the $p$-cymene ligand were modelled as disordered over two orientations with occupancies of 0.561 (15) and 0.439 (15).

\section{Acknowledgements}

We gratefully acknowledge Anglo American Technical Solutions: Research for generous donations of potassium osmate. We would like to thank the Molecular Sciences Institute at the University of the Witwatersrand for infrastructure support. The National Research Foundation National Equipment Programme (UID: 78572) is thanked for financing the purchase of the single crystal diffractometer.

\section{Funding information}

This work is based on the research supported in part by the National Research Foundation of South Africa, Grant Nos. 99234 and 96345.

\section{References}

Bruijnincx, P. C. A. \& Sadler, P. J. (2009). Adv. Inorg. Chem. 61, 1-62. Bruker (2016). APEX2, SAINT and SADABS. BrukerAXS Inc, Madison, Wisconsin, USA.

Dwivedi, A. D., Binnani, C., Tyagi, D., Rawat, K. S., Li, P.-Z., Zhao, Y., Mobin, S. M., Pathak, B. \& Singh, S. K. (2016). Inorg. Chem. 55, 6739-6749.

Farrugia, L. J. (2012). J. Appl. Cryst. 45, 849-854.

Groom, C. R., Bruno, I. J., Lightfoot, M. P. \& Ward, S. C. (2016). Acta Cryst. B72, 171-179.

Hanif, M., Babak, M. V. \& Hartinger, C. G. (2014). Drug Discov. Today, 19, 1640-1648.

Hanif, M., Henke, H., Meier, S. M., Martic, S., Labib, M., Kandioller, W., Jakupec, M. A., Arion, V. B., Kraatz, H.-B., Keppler, B. K. \& Hartinger, C. G. (2010). Inorg. Chem. 49, 7953-7963.

Kandioller, W., Balsano, E., Meier, S. M., Jungwirth, U., Göschl, S., Roller, A., Jakupec, M. A., Berger, W., Keppler, B. K. \& Hartinger, C. G. (2013). Chem. Commun. 49, 3348-3350.

Peacock, A. F. A., Melchart, M., Deeth, R. J., Habtemariam, A., Parsons, S. \& Sadler, P. J. (2007). Chem. Eur. J. 13, 2601-2613.

Rijt, S. H. van, Peacock, A. F., Johnstone, R. D., Parsons, S. \& Sadler, P. J. (2009). Inorg. Chem. 48, 1753-1762.

Sheldrick, G. M. (2008). Acta Cryst. A64, 112-122.

Sheldrick, G. M. (2015). Acta Cryst. C71, 3-8.

Zhang, P. \& Sadler, P. J. (2017). J. Organomet. Chem. 839, 5-14. 


\section{supporting information}

Acta Cryst. (2018). E74, 275-277 [https://doi.org/10.1107/S2056989018001391]

\section{Crystal structure of bromido( $\eta^{6}$-1-isopropyl-4-methylbenzene)(7-oxocyclo-} hepta-1,3,5-trien-1-olato- $\left.\kappa^{2} O, O^{\prime}\right)$ osmium

\section{Hadley S. Clayton, Kgaugelo C. Tapala and Andreas Lemmerer}

Computing details

Data collection: APEX3 (Bruker, 2016); cell refinement: SAINT-Plus (Bruker, 2016); data reduction: SAINT-Plus and XPREP (Bruker, 2016); program(s) used to solve structure: SHELXS97 (Sheldrick, 2008); program(s) used to refine structure: SHELXL2014/7 (Sheldrick, 2015); molecular graphics: ORTEP for Windows (Farrugia, 2012); software used to prepare material for publication: Win $G X$ publication routines (Farrugia, 2012).

Bromido $\left(\eta^{6}\right.$-1-isopropyl-4-methylbenzene)(7-oxocyclohepta-1,3,5-trien- $\backslash 1$-olato- $\left.\kappa^{2} O, O^{\prime}\right)$ osmium

Crystal data

$\mathrm{C}_{17} \mathrm{H}_{19} \mathrm{BrO}_{2} \mathrm{Os}$

$M_{r}=525.43$

Monoclinic, $P 2_{1} / c$

Hall symbol: -P 2ybc

$a=11.1574(5) \AA$

$b=14.6104(7) \AA$

$c=10.8342(5) \AA$

$\beta=110.454(2)^{\circ}$

$V=1654.78(13) \AA^{3}$

$Z=4$

\section{Data collection}

Bruker D8 Venture Photon CCD area detector diffractometer

Graphite monochromator

$\omega$ scans

Absorption correction: integration

Bruker XPREP (Bruker, 2016)

$T_{\min }=0.538, T_{\max }=0.714$

58616 measured reflections

\section{Refinement}

Refinement on $F^{2}$

Least-squares matrix: full

$R\left[F^{2}>2 \sigma\left(F^{2}\right)\right]=0.053$

$w R\left(F^{2}\right)=0.118$

$S=1.15$

3990 reflections

259 parameters

384 restraints

0 constraints
$F(000)=992$

$D_{\mathrm{x}}=2.109 \mathrm{Mg} \mathrm{m}^{-3}$

Mo $K \alpha$ radiation, $\lambda=0.71073 \AA$

Cell parameters from 9941 reflections

$\theta=3.3-28.3^{\circ}$

$\mu=10.12 \mathrm{~mm}^{-1}$

$T=173 \mathrm{~K}$

Block, red

$0.12 \times 0.10 \times 0.05 \mathrm{~mm}$

3990 independent reflections

3614 reflections with $I>2 \sigma(I)$

$R_{\text {int }}=0.079$

$\theta_{\max }=28.0^{\circ}, \theta_{\min }=3.4^{\circ}$

$h=-14 \rightarrow 14$

$k=-19 \rightarrow 19$

$l=-14 \rightarrow 14$

Hydrogen site location: inferred from neighbouring sites

$\mathrm{H}$-atom parameters constrained

$w=1 /\left[\sigma^{2}\left(F_{\mathrm{o}}^{2}\right)+(0.0137 P)^{2}+44.2634 P\right]$

where $P=\left(F_{\mathrm{o}}{ }^{2}+2 F_{\mathrm{c}}{ }^{2}\right) / 3$

$(\Delta / \sigma)_{\max }<0.001$

$\Delta \rho_{\max }=5.63 \mathrm{e} \AA^{-3}$

$\Delta \rho_{\min }=-2.07$ e $\AA^{-3}$ 


\section{Special details}

Experimental. Numerical integration absorption corrections based on indexed crystal faces were applied using the XPREP routine (Bruker, 2016)

Geometry. All esds (except the esd in the dihedral angle between two 1.s. planes) are estimated using the full covariance matrix. The cell esds are taken into account individually in the estimation of esds in distances, angles and torsion angles; correlations between esds in cell parameters are only used when they are defined by crystal symmetry. An approximate (isotropic) treatment of cell esds is used for estimating esds involving l.s. planes.

Fractional atomic coordinates and isotropic or equivalent isotropic displacement parameters $\left(\AA^{2}\right)$

\begin{tabular}{|c|c|c|c|c|c|}
\hline & $x$ & $y$ & $z$ & $U_{\text {iso }} * / U_{\text {eq }}$ & Occ. $(<1)$ \\
\hline C1A & $0.2170(16)$ & $1.0600(9)$ & $-0.1234(11)$ & $0.035(3)$ & $0.561(15)$ \\
\hline $\mathrm{C} 2 \mathrm{~A}$ & $0.1066(14)$ & $1.0131(11)$ & $-0.1297(16)$ & $0.034(3)$ & $0.561(15)$ \\
\hline $\mathrm{H} 2 \mathrm{~A}$ & 0.0364 & 1.0455 & -0.1202 & $0.041 *$ & $0.561(15)$ \\
\hline $\mathrm{C} 3 \mathrm{~A}$ & 0.0988 (13) & $0.9190(11)$ & $-0.1497(16)$ & $0.036(3)$ & $0.561(15)$ \\
\hline $\mathrm{H} 3 \mathrm{~A}$ & 0.0234 & 0.887 & -0.154 & $0.043 *$ & $0.561(15)$ \\
\hline $\mathrm{C} 4 \mathrm{~A}$ & $0.2015(16)$ & $0.8718(9)$ & $-0.1635(12)$ & $0.035(3)$ & $0.561(15)$ \\
\hline C5A & $0.3120(14)$ & $0.9186(13)$ & $-0.1572(19)$ & $0.034(3)$ & $0.561(15)$ \\
\hline $\mathrm{H} 5 \mathrm{~A}$ & 0.3822 & 0.8863 & -0.1666 & $0.041 *$ & $0.561(15)$ \\
\hline C6A & 0.3197 (13) & $1.0127(13)$ & $-0.1372(19)$ & $0.033(3)$ & $0.561(15)$ \\
\hline H6A & 0.3951 & 1.0447 & -0.1329 & $0.039 *$ & $0.561(15)$ \\
\hline C7A & $0.2286(19)$ & $1.1633(10)$ & $-0.1010(16)$ & $0.040(3)$ & $0.561(15)$ \\
\hline H7A & 0.3211 & 1.1768 & -0.0529 & $0.048 *$ & $0.561(15)$ \\
\hline C8A & $0.155(2)$ & 1.1965 (17) & $-0.013(2)$ & $0.050(5)$ & $0.561(15)$ \\
\hline H8A1 & 0.1833 & 1.1617 & 0.0691 & $0.076^{*}$ & $0.561(15)$ \\
\hline H8A2 & 0.1726 & 1.2617 & 0.0059 & $0.076^{*}$ & $0.561(15)$ \\
\hline H8A3 & 0.0635 & 1.1873 & -0.0592 & $0.076^{*}$ & $0.561(15)$ \\
\hline C9A & $0.190(2)$ & $1.2146(16)$ & $-0.2316(19)$ & $0.048(5)$ & $0.561(15)$ \\
\hline H9A1 & 0.1982 & 1.2806 & -0.2148 & $0.073 *$ & $0.561(15)$ \\
\hline H9A2 & 0.246 & 1.1962 & -0.2797 & $0.073^{*}$ & $0.561(15)$ \\
\hline H9A3 & 0.1011 & 1.1999 & -0.2844 & $0.073 *$ & $0.561(15)$ \\
\hline $\mathrm{C} 10 \mathrm{~A}$ & $0.193(2)$ & $0.7683(12)$ & $-0.1851(17)$ & $0.046(4)$ & $0.561(15)$ \\
\hline H10A & 0.144 & 0.7411 & -0.1345 & $0.068 *$ & $0.561(15)$ \\
\hline H10B & 0.2789 & 0.742 & -0.1558 & $0.068^{*}$ & $0.561(15)$ \\
\hline $\mathrm{H} 10 \mathrm{C}$ & 0.1493 & 0.7552 & -0.279 & $0.068^{*}$ & $0.561(15)$ \\
\hline C1B & $0.175(2)$ & $0.8944(11)$ & $-0.1821(12)$ & $0.033(3)$ & $0.439(15)$ \\
\hline $\mathrm{C} 2 \mathrm{~B}$ & $0.0910(16)$ & $0.9528(13)$ & $-0.1515(19)$ & $0.028(3)$ & $0.439(15)$ \\
\hline $\mathrm{H} 2 \mathrm{~B}$ & 0.0097 & 0.931 & -0.1548 & $0.034 *$ & $0.439(15)$ \\
\hline $\mathrm{C} 3 \mathrm{~B}$ & $0.1259(18)$ & $1.0430(12)$ & $-0.116(2)$ & $0.031(4)$ & $0.439(15)$ \\
\hline H3B & 0.0684 & 1.0829 & -0.0953 & $0.037 *$ & $0.439(15)$ \\
\hline $\mathrm{C} 4 \mathrm{~B}$ & 0.2448 (19) & 1.0749 (12) & $-0.1114(16)$ & $0.031(3)$ & $0.439(15)$ \\
\hline C5B & $0.3288(16)$ & $1.0166(17)$ & $-0.142(2)$ & $0.028(3)$ & $0.439(15)$ \\
\hline H5B & 0.4101 & 1.0384 & -0.1387 & $0.033^{*}$ & $0.439(15)$ \\
\hline C6B & 0.2939 (19) & $0.9263(15)$ & $-0.177(2)$ & $0.031(3)$ & $0.439(15)$ \\
\hline H6B & 0.3514 & 0.8865 & -0.1982 & $0.037 *$ & $0.439(15)$ \\
\hline $\mathrm{C} 7 \mathrm{~B}$ & $0.140(2)$ & $0.7948(13)$ & $-0.2213(16)$ & $0.042(4)$ & $0.439(15)$ \\
\hline H7B & 0.2226 & 0.7599 & -0.1945 & $0.05 *$ & $0.439(15)$ \\
\hline $\mathrm{C} 8 \mathrm{~B}$ & $0.054(3)$ & $0.7458(19)$ & -0.160 & $0.049(6)$ & $0.439(15)$ \\
\hline
\end{tabular}




\begin{tabular}{|c|c|c|c|c|c|}
\hline H8B1 & 0.0927 & 0.7492 & -0.0633 & $0.073 *$ & $0.439(15)$ \\
\hline H8B2 & -0.03 & 0.7752 & -0.1885 & $0.073 *$ & $0.439(15)$ \\
\hline H8B3 & 0.0447 & 0.6815 & -0.1871 & $0.073 *$ & $0.439(15)$ \\
\hline C9B & $0.084(3)$ & $0.791(2)$ & $-0.3714(18)$ & $0.047(6)$ & $0.439(15)$ \\
\hline H9B1 & 0.1405 & 0.8228 & -0.4086 & $0.07 *$ & $0.439(15)$ \\
\hline H9B2 & 0.0743 & 0.727 & -0.4003 & $0.07^{*}$ & $0.439(15)$ \\
\hline H9B3 & -0.0004 & 0.8207 & -0.4018 & $0.07^{*}$ & $0.439(15)$ \\
\hline C10B & $0.280(3)$ & $1.1744(14)$ & $-0.072(2)$ & $0.040(5)$ & $0.439(15)$ \\
\hline H10D & 0.2606 & 1.2121 & -0.1511 & $0.06^{*}$ & $0.439(15)$ \\
\hline H10E & 0.3709 & 1.1786 & -0.0195 & $0.06^{*}$ & $0.439(15)$ \\
\hline $\mathrm{H} 10 \mathrm{~F}$ & 0.2294 & 1.1963 & -0.0193 & $0.06^{*}$ & $0.439(15)$ \\
\hline C11 & $0.2519(8)$ & $0.9918(7)$ & $0.2698(9)$ & $0.0254(18)$ & \\
\hline $\mathrm{C} 12$ & $0.2049(9)$ & $0.9871(8)$ & $0.3746(10)$ & $0.033(2)$ & \\
\hline H12 & 0.1296 & 0.9515 & 0.3575 & $0.04^{*}$ & \\
\hline C13 & $0.2518(10)$ & $1.0265(8)$ & $0.4988(10)$ & $0.037(2)$ & \\
\hline H13 & 0.2066 & 1.0116 & 0.5559 & $0.045^{*}$ & \\
\hline $\mathrm{C} 14$ & $0.3564(11)$ & $1.0851(9)$ & $0.5517(11)$ & $0.045(3)$ & \\
\hline H14 & 0.3716 & 1.1058 & 0.639 & $0.054 *$ & \\
\hline $\mathrm{C} 15$ & $0.4393(10)$ & $1.1163(8)$ & $0.4942(10)$ & $0.038(2)$ & \\
\hline H15 & 0.5031 & 1.1574 & 0.5459 & $0.045^{*}$ & \\
\hline $\mathrm{C} 16$ & $0.4436(9)$ & $1.0967(7)$ & $0.3722(10)$ & $0.032(2)$ & \\
\hline H16 & 0.5106 & 1.1266 & 0.3528 & $0.038 *$ & \\
\hline $\mathrm{C} 17$ & $0.3659(8)$ & $1.0398(7)$ & $0.2702(9)$ & $0.0256(18)$ & \\
\hline $\mathrm{O} 1$ & $0.1888(6)$ & $0.9495(5)$ & $0.1605(6)$ & $0.0312(15)$ & \\
\hline $\mathrm{O} 2$ & $0.3948(6)$ & $1.0300(5)$ & 0.1647 (6) & $0.0287(14)$ & \\
\hline $\mathrm{Br} 1$ & $0.40095(10)$ & $0.81751(8)$ & $0.13655(12)$ & $0.0417(3)$ & \\
\hline Os1 & $0.26979(3)$ & 0.95325 & $0.01352(3)$ & $0.02377(12)$ & \\
\hline
\end{tabular}

Atomic displacement parameters $\left(\AA^{2}\right)$

\begin{tabular}{lllllll}
\hline & $U^{11}$ & $U^{22}$ & $U^{33}$ & $U^{12}$ & $U^{13}$ & $U^{23}$ \\
\hline C1A & $0.019(6)$ & $0.056(6)$ & $0.031(6)$ & $0.004(5)$ & $0.011(5)$ & $0.007(5)$ \\
C2A & $0.018(6)$ & $0.061(7)$ & $0.022(6)$ & $-0.002(6)$ & $0.005(5)$ & $0.006(7)$ \\
C3A & $0.019(5)$ & $0.064(8)$ & $0.024(6)$ & $-0.007(6)$ & $0.007(5)$ & $0.001(7)$ \\
C4A & $0.028(6)$ & $0.060(7)$ & $0.015(6)$ & $-0.003(5)$ & $0.007(5)$ & $0.000(6)$ \\
C5A & $0.021(5)$ & $0.059(6)$ & $0.022(7)$ & $0.002(5)$ & $0.008(6)$ & $-0.001(6)$ \\
C6A & $0.025(5)$ & $0.056(5)$ & $0.022(6)$ & $0.002(5)$ & $0.016(5)$ & $0.008(6)$ \\
C7A & $0.029(9)$ & $0.055(6)$ & $0.048(8)$ & $0.000(6)$ & $0.026(7)$ & $0.012(7)$ \\
C8A & $0.058(12)$ & $0.059(11)$ & $0.047(10)$ & $-0.001(10)$ & $0.034(9)$ & $-0.001(9)$ \\
C9A & $0.052(11)$ & $0.056(10)$ & $0.046(9)$ & $0.012(10)$ & $0.028(9)$ & $0.011(8)$ \\
C10A & $0.038(10)$ & $0.065(7)$ & $0.032(9)$ & $-0.009(7)$ & $0.010(8)$ & $-0.012(9)$ \\
C1B & $0.022(7)$ & $0.056(7)$ & $0.017(7)$ & $-0.003(5)$ & $0.002(6)$ & $-0.001(6)$ \\
C2B & $0.022(6)$ & $0.051(8)$ & $0.017(6)$ & $-0.005(6)$ & $0.013(5)$ & $0.013(7)$ \\
C3B & $0.016(6)$ & $0.056(8)$ & $0.022(7)$ & $0.002(6)$ & $0.009(6)$ & $0.000(7)$ \\
C4B & $0.022(7)$ & $0.048(6)$ & $0.028(7)$ & $-0.002(5)$ & $0.015(6)$ & $0.008(6)$ \\
C5B & $0.021(6)$ & $0.049(6)$ & $0.021(7)$ & $0.001(5)$ & $0.017(6)$ & $0.014(6)$ \\
C6B & $0.027(6)$ & $0.056(6)$ & $0.013(7)$ & $0.000(5)$ & $0.012(6)$ & $0.001(6)$ \\
C7B & $0.029(9)$ & $0.059(8)$ & $0.030(8)$ & $-0.004(7)$ & $0.003(7)$ & $-0.006(8)$
\end{tabular}




\begin{tabular}{lllllll} 
C8B & $0.055(14)$ & $0.050(12)$ & $0.044(11)$ & $-0.010(10)$ & $0.020(11)$ & $-0.009(11)$ \\
C9B & $0.050(14)$ & $0.058(14)$ & $0.030(8)$ & $0.005(11)$ & $0.013(9)$ & $-0.013(8)$ \\
C10B & $0.030(11)$ & $0.051(8)$ & $0.047(12)$ & $-0.002(8)$ & $0.023(10)$ & $0.000(9)$ \\
C11 & $0.017(4)$ & $0.038(5)$ & $0.023(4)$ & $0.007(4)$ & $0.009(3)$ & $0.004(4)$ \\
C12 & $0.024(4)$ & $0.050(6)$ & $0.028(5)$ & $0.001(4)$ & $0.012(4)$ & $0.004(4)$ \\
C13 & $0.033(5)$ & $0.060(7)$ & $0.025(5)$ & $0.005(5)$ & $0.018(4)$ & $0.000(4)$ \\
C14 & $0.046(6)$ & $0.057(7)$ & $0.025(5)$ & $0.018(6)$ & $0.002(5)$ & $-0.006(5)$ \\
C15 & $0.037(5)$ & $0.043(6)$ & $0.028(5)$ & $0.003(5)$ & $0.005(4)$ & $-0.006(4)$ \\
C16 & $0.028(5)$ & $0.038(6)$ & $0.029(5)$ & $0.001(4)$ & $0.010(4)$ & $-0.004(4)$ \\
C17 & $0.020(4)$ & $0.033(5)$ & $0.025(4)$ & $0.004(4)$ & $0.009(3)$ & $0.000(4)$ \\
O1 & $0.019(3)$ & $0.053(4)$ & $0.024(3)$ & $-0.005(3)$ & $0.010(3)$ & $-0.006(3)$ \\
O2 & $0.020(3)$ & $0.046(4)$ & $0.026(3)$ & $-0.006(3)$ & $0.016(3)$ & $-0.004(3)$ \\
Br1 & $0.0316(5)$ & $0.0392(6)$ & $0.0526(7)$ & $0.0059(4)$ & $0.0128(5)$ & $0.0038(5)$ \\
Os1 & $0.01538(16)$ & $0.0385(2)$ & $0.02000(17)$ & $0.00078(14)$ & $0.00944(12)$ & $-0.00243(15)$ \\
& & & & & & \\
\hline
\end{tabular}

Geometric parameters $\left(A,{ }^{\circ}\right)$

\begin{tabular}{|c|c|c|c|}
\hline Os1-C1A & $2.090(12)$ & $\mathrm{C} 1 \mathrm{~B}-\mathrm{C} 2 \mathrm{~B}$ & 1.39 \\
\hline Os1- $22 \mathrm{~A}$ & $2.125(14)$ & $\mathrm{C} 1 \mathrm{~B}-\mathrm{C} 6 \mathrm{~B}$ & 1.39 \\
\hline Os1-C3A & $2.158(14)$ & $\mathrm{C} 1 \mathrm{~B}-\mathrm{C} 7 \mathrm{~B}$ & $1.528(10)$ \\
\hline Os1-C4A & $2.157(12)$ & $\mathrm{C} 2 \mathrm{~B}-\mathrm{C} 3 \mathrm{~B}$ & 1.39 \\
\hline Os1-C $5 \mathrm{~A}$ & $2.123(18)$ & $\mathrm{C} 2 \mathrm{~B}-\mathrm{H} 2 \mathrm{~B}$ & 0.95 \\
\hline Os1-C6A & $2.089(18)$ & $\mathrm{C} 3 \mathrm{~B}-\mathrm{C} 4 \mathrm{~B}$ & 1.39 \\
\hline Os1-C1B & 2.187 (13) & $\mathrm{C} 3 \mathrm{~B}-\mathrm{H} 3 \mathrm{~B}$ & 0.95 \\
\hline Os1-C2B & $2.164(17)$ & $\mathrm{C} 4 \mathrm{~B}-\mathrm{C} 5 \mathrm{~B}$ & 1.39 \\
\hline Os1-C3B & 2.167 (19) & $\mathrm{C} 4 \mathrm{~B}-\mathrm{C} 10 \mathrm{~B}$ & $1.526(10)$ \\
\hline Os1-C4B & $2.192(16)$ & $\mathrm{C} 5 \mathrm{~B}-\mathrm{C} 6 \mathrm{~B}$ & 1.39 \\
\hline Os1-C5B & $2.21(2)$ & $\mathrm{C} 5 \mathrm{~B}-\mathrm{H} 5 \mathrm{~B}$ & 0.95 \\
\hline Os1-C6B & $2.21(2)$ & $\mathrm{C} 6 \mathrm{~B}-\mathrm{H} 6 \mathrm{~B}$ & 0.95 \\
\hline Os1-O1 & $2.088(6)$ & $\mathrm{C} 7 \mathrm{~B}-\mathrm{C} 9 \mathrm{~B}$ & $1.527(11)$ \\
\hline $\mathrm{Os} 1-\mathrm{O} 2$ & $2.071(6)$ & $\mathrm{C} 7 \mathrm{~B}-\mathrm{C} 8 \mathrm{~B}$ & $1.527(11)$ \\
\hline Os1-Br1 & $2.5472(12)$ & $\mathrm{C} 7 \mathrm{~B}-\mathrm{H} 7 \mathrm{~B}$ & 1 \\
\hline $\mathrm{C} 1 \mathrm{~A}-\mathrm{C} 2 \mathrm{~A}$ & 1.39 & $\mathrm{C} 8 \mathrm{~B}-\mathrm{H} 8 \mathrm{~B} 1$ & 0.98 \\
\hline $\mathrm{C} 1 \mathrm{~A}-\mathrm{C} 6 \mathrm{~A}$ & 1.39 & $\mathrm{C} 8 \mathrm{~B}-\mathrm{H} 8 \mathrm{~B} 2$ & 0.98 \\
\hline $\mathrm{C} 1 \mathrm{~A}-\mathrm{C} 7 \mathrm{~A}$ & $1.528(10)$ & $\mathrm{C} 8 \mathrm{~B}-\mathrm{H} 8 \mathrm{~B} 3$ & 0.98 \\
\hline $\mathrm{C} 2 \mathrm{~A}-\mathrm{C} 3 \mathrm{~A}$ & 1.39 & C9B-H9B1 & 0.98 \\
\hline $\mathrm{C} 2 \mathrm{~A}-\mathrm{H} 2 \mathrm{~A}$ & 0.95 & $\mathrm{C} 9 \mathrm{~B}-\mathrm{H} 9 \mathrm{~B} 2$ & 0.98 \\
\hline $\mathrm{C} 3 \mathrm{~A}-\mathrm{C} 4 \mathrm{~A}$ & 1.39 & C9B-H9B3 & 0.98 \\
\hline $\mathrm{C} 3 \mathrm{~A}-\mathrm{H} 3 \mathrm{~A}$ & 0.95 & $\mathrm{C} 10 \mathrm{~B}-\mathrm{H} 10 \mathrm{D}$ & 0.98 \\
\hline $\mathrm{C} 4 \mathrm{~A}-\mathrm{C} 5 \mathrm{~A}$ & 1.39 & $\mathrm{C} 10 \mathrm{~B}-\mathrm{H} 10 \mathrm{E}$ & 0.98 \\
\hline $\mathrm{C} 4 \mathrm{~A}-\mathrm{C} 10 \mathrm{~A}$ & $1.528(10)$ & $\mathrm{C} 10 \mathrm{~B}-\mathrm{H} 10 \mathrm{~F}$ & 0.98 \\
\hline $\mathrm{C} 5 \mathrm{~A}-\mathrm{C} 6 \mathrm{~A}$ & 1.39 & $\mathrm{C} 11-\mathrm{O} 1$ & $1.303(11)$ \\
\hline $\mathrm{C} 5 \mathrm{~A}-\mathrm{H} 5 \mathrm{~A}$ & 0.95 & $\mathrm{C} 11-\mathrm{C} 12$ & $1.408(13)$ \\
\hline $\mathrm{C} 6 \mathrm{~A}-\mathrm{H} 6 \mathrm{~A}$ & 0.95 & $\mathrm{C} 11-\mathrm{C} 17$ & $1.452(13)$ \\
\hline $\mathrm{C} 7 \mathrm{~A}-\mathrm{C} 9 \mathrm{~A}$ & $1.524(10)$ & $\mathrm{C} 12-\mathrm{C} 13$ & $1.388(14)$ \\
\hline $\mathrm{C} 7 \mathrm{~A}-\mathrm{C} 8 \mathrm{~A}$ & $1.531(10)$ & $\mathrm{C} 12-\mathrm{H} 12$ & 0.95 \\
\hline $\mathrm{C} 7 \mathrm{~A}-\mathrm{H} 7 \mathrm{~A}$ & 1 & $\mathrm{C} 13-\mathrm{C} 14$ & $1.399(17)$ \\
\hline $\mathrm{C} 8 \mathrm{~A}-\mathrm{H} 8 \mathrm{~A} 1$ & 0.98 & $\mathrm{C} 13-\mathrm{H} 13$ & 0.95 \\
\hline
\end{tabular}


C8A-H8A2
C8A-H8A3
C9A-H9A1
C9A-H9A2
C9A-H9A3
C10A-H10A
C10A-H10B
C10A-H10C

$\mathrm{C} 2 \mathrm{~A}-\mathrm{C} 1 \mathrm{~A}-\mathrm{C} 6 \mathrm{~A}$

$\mathrm{C} 2 \mathrm{~A}-\mathrm{C} 1 \mathrm{~A}-\mathrm{C} 7 \mathrm{~A}$

$\mathrm{C} 6 \mathrm{~A}-\mathrm{C} 1 \mathrm{~A}-\mathrm{C} 7 \mathrm{~A}$

$\mathrm{C} 2 \mathrm{~A}-\mathrm{C} 1 \mathrm{~A}-\mathrm{Os} 1$

C6A-C1A-Os1

$\mathrm{C} 7 \mathrm{~A}-\mathrm{C} 1 \mathrm{~A}-\mathrm{Os} 1$

$\mathrm{C} 1 \mathrm{~A}-\mathrm{C} 2 \mathrm{~A}-\mathrm{C} 3 \mathrm{~A}$

$\mathrm{C} 1 \mathrm{~A}-\mathrm{C} 2 \mathrm{~A}-\mathrm{Os} 1$

$\mathrm{C} 3 \mathrm{~A}-\mathrm{C} 2 \mathrm{~A}-\mathrm{Os} 1$

$\mathrm{C} 1 \mathrm{~A}-\mathrm{C} 2 \mathrm{~A}-\mathrm{H} 2 \mathrm{~A}$

$\mathrm{C} 3 \mathrm{~A}-\mathrm{C} 2 \mathrm{~A}-\mathrm{H} 2 \mathrm{~A}$

$\mathrm{O} 1-\mathrm{C} 2 \mathrm{~A}-\mathrm{H} 2 \mathrm{~A}$

$\mathrm{C} 2 \mathrm{~A}-\mathrm{C} 3 \mathrm{~A}-\mathrm{C} 4 \mathrm{~A}$

$\mathrm{C} 2 \mathrm{~A}-\mathrm{C} 3 \mathrm{~A}-\mathrm{Os} 1$

$\mathrm{C} 4 \mathrm{~A}-\mathrm{C} 3 \mathrm{~A}-\mathrm{Os} 1$

$\mathrm{C} 2 \mathrm{~A}-\mathrm{C} 3 \mathrm{~A}-\mathrm{H} 3 \mathrm{~A}$

$\mathrm{C} 4 \mathrm{~A}-\mathrm{C} 3 \mathrm{~A}-\mathrm{H} 3 \mathrm{~A}$

Os1-C3A-H3A

$\mathrm{C} 5 \mathrm{~A}-\mathrm{C} 4 \mathrm{~A}-\mathrm{C} 3 \mathrm{~A}$

$\mathrm{C} 5 \mathrm{~A}-\mathrm{C} 4 \mathrm{~A}-\mathrm{C} 10 \mathrm{~A}$

$\mathrm{C} 3 \mathrm{~A}-\mathrm{C} 4 \mathrm{~A}-\mathrm{C} 10 \mathrm{~A}$

$\mathrm{C} 5 \mathrm{~A}-\mathrm{C} 4 \mathrm{~A}-\mathrm{Os} 1$

$\mathrm{C} 3 \mathrm{~A}-\mathrm{C} 4 \mathrm{~A}-\mathrm{Os} 1$

$\mathrm{C} 10 \mathrm{~A}-\mathrm{C} 4 \mathrm{~A}-\mathrm{Os} 1$

$\mathrm{C} 6 \mathrm{~A}-\mathrm{C} 5 \mathrm{~A}-\mathrm{C} 4 \mathrm{~A}$

C6A-C5A-Os1

$\mathrm{C} 4 \mathrm{~A}-\mathrm{C} 5 \mathrm{~A}-\mathrm{Os} 1$

$\mathrm{C} 6 \mathrm{~A}-\mathrm{C} 5 \mathrm{~A}-\mathrm{H} 5 \mathrm{~A}$

$\mathrm{C} 4 \mathrm{~A}-\mathrm{C} 5 \mathrm{~A}-\mathrm{H} 5 \mathrm{~A}$

Os1-C $5 \mathrm{~A}-\mathrm{H} 5 \mathrm{~A}$

$\mathrm{C} 5 \mathrm{~A}-\mathrm{C} 6 \mathrm{~A}-\mathrm{C} 1 \mathrm{~A}$

$\mathrm{C} 5 \mathrm{~A}-\mathrm{C} 6 \mathrm{~A}-\mathrm{Os} 1$

$\mathrm{C} 1 \mathrm{~A}-\mathrm{C} 6 \mathrm{~A}-\mathrm{Os} 1$

C5A-C6A-H6A

$\mathrm{C} 1 \mathrm{~A}-\mathrm{C} 6 \mathrm{~A}-\mathrm{H} 6 \mathrm{~A}$

Os1-C6A-H6A

$\mathrm{C} 9 \mathrm{~A}-\mathrm{C} 7 \mathrm{~A}-\mathrm{C} 1 \mathrm{~A}$

C9A-C7A-C8A

$\mathrm{C} 1 \mathrm{~A}-\mathrm{C} 7 \mathrm{~A}-\mathrm{C} 8 \mathrm{~A}$
0.98

0.98

0.98

0.98

0.98

0.98

0.98

0.98

120

$121.3(12)$

$118.7(12)$

$72.1(6)$

70.5 (6)

$129.6(4)$

120

69.4 (6)

72.4 (4)

120

120

130.9

120

69.8 (4)

$71.2(5)$

120

120

131.9

120

$120.2(13)$

119.8 (13)

69.7 (6)

$71.2(6)$

$131.8(4)$

120

69.4 (4)

72.4 (5)

120

120

130.9

120

72.1 (4)

$70.6(5)$

120

120

129.8

110.9 (12)

$112.4(16)$

$112.2(15)$
C14-C15

C14-H14

C15-C16

C15-H15

C16-C17

C16- 1616

C17-O2

$\mathrm{C} 5 \mathrm{~B}-\mathrm{C} 6 \mathrm{~B}-\mathrm{H} 6 \mathrm{~B}$

$\mathrm{C} 1 \mathrm{~B}-\mathrm{C} 6 \mathrm{~B}-\mathrm{H} 6 \mathrm{~B}$

Os1- C6B-H6B

$\mathrm{C} 9 \mathrm{~B}-\mathrm{C} 7 \mathrm{~B}-\mathrm{C} 8 \mathrm{~B}$

$\mathrm{C} 9 \mathrm{~B}-\mathrm{C} 7 \mathrm{~B}-\mathrm{C} 1 \mathrm{~B}$

$\mathrm{C} 8 \mathrm{~B}-\mathrm{C} 7 \mathrm{~B}-\mathrm{C} 1 \mathrm{~B}$

$\mathrm{C} 9 \mathrm{~B}-\mathrm{C} 7 \mathrm{~B}-\mathrm{H} 7 \mathrm{~B}$

$\mathrm{C} 8 \mathrm{~B}-\mathrm{C} 7 \mathrm{~B}-\mathrm{H} 7 \mathrm{~B}$

$\mathrm{C} 1 \mathrm{~B}-\mathrm{C} 7 \mathrm{~B}-\mathrm{H} 7 \mathrm{~B}$

$\mathrm{C} 7 \mathrm{~B}-\mathrm{C} 8 \mathrm{~B}-\mathrm{H} 8 \mathrm{~B} 1$

$\mathrm{C} 7 \mathrm{~B}-\mathrm{C} 8 \mathrm{~B}-\mathrm{H} 8 \mathrm{~B} 2$

$\mathrm{H} 8 \mathrm{~B} 1-\mathrm{C} 8 \mathrm{~B}-\mathrm{H} 8 \mathrm{~B} 2$

$\mathrm{C} 7 \mathrm{~B}-\mathrm{C} 8 \mathrm{~B}-\mathrm{H} 8 \mathrm{~B} 3$

$\mathrm{H} 8 \mathrm{~B} 1-\mathrm{C} 8 \mathrm{~B}-\mathrm{H} 8 \mathrm{~B} 3$

$\mathrm{H} 8 \mathrm{~B} 2-\mathrm{C} 8 \mathrm{~B}-\mathrm{H} 8 \mathrm{~B} 3$

$\mathrm{C} 7 \mathrm{~B}-\mathrm{C} 9 \mathrm{~B}-\mathrm{H} 9 \mathrm{~B} 1$

$\mathrm{C} 7 \mathrm{~B}-\mathrm{C} 9 \mathrm{~B}-\mathrm{H} 9 \mathrm{~B} 2$

$\mathrm{H} 9 \mathrm{~B} 1-\mathrm{C} 9 \mathrm{~B}-\mathrm{H} 9 \mathrm{~B} 2$

$\mathrm{C} 7 \mathrm{~B}-\mathrm{C} 9 \mathrm{~B}-\mathrm{H} 9 \mathrm{~B} 3$

H9B $1-C 9 B-H 9 B 3$

H9B2 - C9B-H9B3

$\mathrm{C} 4 \mathrm{~B}-\mathrm{C} 10 \mathrm{~B}-\mathrm{H} 10 \mathrm{D}$

$\mathrm{C} 4 \mathrm{~B}-\mathrm{C} 10 \mathrm{~B}-\mathrm{H} 10 \mathrm{E}$

H10D-C10B-H10E

$\mathrm{C} 4 \mathrm{~B}-\mathrm{C} 10 \mathrm{~B}-\mathrm{H} 10 \mathrm{~F}$

H10D-C10B-H10F

H10E-C10B-H10F

$\mathrm{O} 1-\mathrm{C} 11-\mathrm{C} 12$

$\mathrm{O} 1-\mathrm{C} 11-\mathrm{C} 17$

$\mathrm{C} 12-\mathrm{C} 11-\mathrm{C} 17$

$\mathrm{C} 13-\mathrm{C} 12-\mathrm{C} 11$

C13-C12-H12

C11-C12-H12

$\mathrm{C} 12-\mathrm{C} 13-\mathrm{C} 14$

C12-C13-H13

$\mathrm{C} 14-\mathrm{C} 13-\mathrm{H} 13$

C15-C14-C13

$\mathrm{C} 15-\mathrm{C} 14-\mathrm{H} 14$

C13-C14-H14
1.362 (17)

0.95

1.369 (14)

0.95

1.413 (13)

0.95

$1.299(11)$

120

120

130.1

$111(2)$

$107.5(14)$

117.5 (19)

106.7

106.7

106.7

109.5

109.5

109.5

109.5

109.5

109.5

109.5

109.5

109.5

109.5

109.5

109.5

109.5

109.5

109.5

109.5

109.5

109.5

118.3 (9)

$115.2(8)$

$126.4(9)$

129.9 (10)

115.1

115.1

129.0 (10)

115.5

115.5

128.3 (10)

115.8

115.8 


\begin{tabular}{|c|c|c|c|}
\hline $\mathrm{C} 9 \mathrm{~A}-\mathrm{C} 7 \mathrm{~A}-\mathrm{H} 7 \mathrm{~A}$ & 107 & $\mathrm{C} 14-\mathrm{C} 15-\mathrm{C} 16$ & $129.1(11)$ \\
\hline $\mathrm{C} 1 \mathrm{~A}-\mathrm{C} 7 \mathrm{~A}-\mathrm{H} 7 \mathrm{~A}$ & 107 & $\mathrm{C} 14-\mathrm{C} 15-\mathrm{H} 15$ & 115.5 \\
\hline $\mathrm{C} 8 \mathrm{~A}-\mathrm{C} 7 \mathrm{~A}-\mathrm{H} 7 \mathrm{~A}$ & 107 & $\mathrm{C} 16-\mathrm{C} 15-\mathrm{H} 15$ & 115.5 \\
\hline $\mathrm{C} 7 \mathrm{~A}-\mathrm{C} 8 \mathrm{~A}-\mathrm{H} 8 \mathrm{~A} 1$ & 109.5 & $\mathrm{C} 15-\mathrm{C} 16-\mathrm{C} 17$ & $131.2(10)$ \\
\hline $\mathrm{C} 7 \mathrm{~A}-\mathrm{C} 8 \mathrm{~A}-\mathrm{H} 8 \mathrm{~A} 2$ & 109.5 & $\mathrm{C} 15-\mathrm{C} 16-\mathrm{H} 16$ & 114.4 \\
\hline $\mathrm{H} 8 \mathrm{~A} 1-\mathrm{C} 8 \mathrm{~A}-\mathrm{H} 8 \mathrm{~A} 2$ & 109.5 & $\mathrm{C} 17-\mathrm{C} 16-\mathrm{H} 16$ & 114.4 \\
\hline $\mathrm{C} 7 \mathrm{~A}-\mathrm{C} 8 \mathrm{~A}-\mathrm{H} 8 \mathrm{~A} 3$ & 109.5 & $\mathrm{O} 2-\mathrm{C} 17-\mathrm{C} 16$ & $118.7(8)$ \\
\hline $\mathrm{H} 8 \mathrm{~A} 1-\mathrm{C} 8 \mathrm{~A}-\mathrm{H} 8 \mathrm{~A} 3$ & 109.5 & $\mathrm{O} 2-\mathrm{C} 17-\mathrm{C} 11$ & $115.5(8)$ \\
\hline $\mathrm{H} 8 \mathrm{~A} 2-\mathrm{C} 8 \mathrm{~A}-\mathrm{H} 8 \mathrm{~A} 3$ & 109.5 & $\mathrm{C} 16-\mathrm{C} 17-\mathrm{C} 11$ & $125.8(9)$ \\
\hline C7A-C9A-H9A1 & 109.5 & $\mathrm{C} 11-\mathrm{O} 1-\mathrm{Os} 1$ & $116.1(6)$ \\
\hline $\mathrm{C} 7 \mathrm{~A}-\mathrm{C} 9 \mathrm{~A}-\mathrm{H} 9 \mathrm{~A} 2$ & 109.5 & $\mathrm{C} 17-\mathrm{O} 2-\mathrm{Os} 1$ & $116.7(6)$ \\
\hline $\mathrm{H} 9 \mathrm{~A} 1-\mathrm{C} 9 \mathrm{~A}-\mathrm{H} 9 \mathrm{~A} 2$ & 109.5 & $\mathrm{O} 2-\mathrm{Os} 1-\mathrm{O} 1$ & $76.3(2)$ \\
\hline C7A-C9A-H9A3 & 109.5 & $\mathrm{O} 2-\mathrm{Os} 1-\mathrm{C} 6 \mathrm{~A}$ & $95.9(5)$ \\
\hline $\mathrm{H} 9 \mathrm{~A} 1-\mathrm{C} 9 \mathrm{~A}-\mathrm{H} 9 \mathrm{~A} 3$ & 109.5 & $\mathrm{O} 1-\mathrm{Os} 1-\mathrm{C} 6 \mathrm{~A}$ & $155.7(6)$ \\
\hline $\mathrm{H} 9 \mathrm{~A} 2-\mathrm{C} 9 \mathrm{~A}-\mathrm{H} 9 \mathrm{~A} 3$ & 109.5 & $\mathrm{O} 2-\mathrm{Os} 1-\mathrm{C} 1 \mathrm{~A}$ & $95.9(4)$ \\
\hline $\mathrm{C} 4 \mathrm{~A}-\mathrm{C} 10 \mathrm{~A}-\mathrm{H} 10 \mathrm{~A}$ & 109.5 & $\mathrm{O} 1-\mathrm{Os} 1-\mathrm{C} 1 \mathrm{~A}$ & $118.1(5)$ \\
\hline $\mathrm{C} 4 \mathrm{~A}-\mathrm{C} 10 \mathrm{~A}-\mathrm{H} 10 \mathrm{~B}$ & 109.5 & $\mathrm{C} 6 \mathrm{~A}-\mathrm{Os} 1-\mathrm{C} 1 \mathrm{~A}$ & $38.9(3)$ \\
\hline $\mathrm{H} 10 \mathrm{~A}-\mathrm{C} 10 \mathrm{~A}-\mathrm{H} 10 \mathrm{~B}$ & 109.5 & $\mathrm{O} 2-\mathrm{Os} 1-\mathrm{C} 5 \mathrm{~A}$ & $121.7(5)$ \\
\hline $\mathrm{C} 4 \mathrm{~A}-\mathrm{C} 10 \mathrm{~A}-\mathrm{H} 10 \mathrm{C}$ & 109.5 & $\mathrm{O} 1-\mathrm{Os} 1-\mathrm{C} 5 \mathrm{~A}$ & $160.9(6)$ \\
\hline $\mathrm{H} 10 \mathrm{~A}-\mathrm{C} 10 \mathrm{~A}-\mathrm{H} 10 \mathrm{C}$ & 109.5 & $\mathrm{C} 6 \mathrm{~A}-\mathrm{Os} 1-\mathrm{C} 5 \mathrm{~A}$ & $38.5(3)$ \\
\hline $\mathrm{H} 10 \mathrm{~B}-\mathrm{C} 10 \mathrm{~A}-\mathrm{H} 10 \mathrm{C}$ & 109.5 & $\mathrm{C} 1 \mathrm{~A}-\mathrm{Os} 1-\mathrm{C} 5 \mathrm{~A}$ & $69.7(4)$ \\
\hline $\mathrm{C} 2 \mathrm{~B}-\mathrm{C} 1 \mathrm{~B}-\mathrm{C} 6 \mathrm{~B}$ & 120 & $\mathrm{O} 2-\mathrm{Os} 1-\mathrm{C} 2 \mathrm{~A}$ & $121.5(5)$ \\
\hline $\mathrm{C} 2 \mathrm{~B}-\mathrm{C} 1 \mathrm{~B}-\mathrm{C} 7 \mathrm{~B}$ & $121.6(16)$ & $\mathrm{O} 1-\mathrm{Os} 1-\mathrm{C} 2 \mathrm{~A}$ & $94.5(5)$ \\
\hline $\mathrm{C} 6 \mathrm{~B}-\mathrm{C} 1 \mathrm{~B}-\mathrm{C} 7 \mathrm{~B}$ & $118.4(16)$ & $\mathrm{C} 6 \mathrm{~A}-\mathrm{Os} 1-\mathrm{C} 2 \mathrm{~A}$ & $69.7(3)$ \\
\hline $\mathrm{C} 2 \mathrm{~B}-\mathrm{C} 1 \mathrm{~B}-\mathrm{Os} 1$ & $70.5(7)$ & $\mathrm{C} 1 \mathrm{~A}-\mathrm{Os} 1-\mathrm{C} 2 \mathrm{~A}$ & $38.5(2)$ \\
\hline $\mathrm{C} 6 \mathrm{~B}-\mathrm{C} 1 \mathrm{~B}-\mathrm{Os} 1$ & $72.5(8)$ & $\mathrm{C} 5 \mathrm{~A}-\mathrm{Os} 1-\mathrm{C} 2 \mathrm{~A}$ & $81.8(3)$ \\
\hline $\mathrm{C} 7 \mathrm{~B}-\mathrm{C} 1 \mathrm{~B}-\mathrm{Os} 1$ & $129.3(5)$ & $\mathrm{O} 2-\mathrm{Os} 1-\mathrm{C} 4 \mathrm{~A}$ & $158.9(5)$ \\
\hline $\mathrm{C} 1 \mathrm{~B}-\mathrm{C} 2 \mathrm{~B}-\mathrm{C} 3 \mathrm{~B}$ & 120 & $\mathrm{O} 1-\mathrm{Os} 1-\mathrm{C} 4 \mathrm{~A}$ & $123.4(5)$ \\
\hline $\mathrm{C} 1 \mathrm{~B}-\mathrm{C} 2 \mathrm{~B}-\mathrm{Os} 1$ & $72.2(7)$ & $\mathrm{C} 6 \mathrm{~A}-\mathrm{Os} 1-\mathrm{C} 4 \mathrm{~A}$ & $69.0(4)$ \\
\hline $\mathrm{C} 3 \mathrm{~B}-\mathrm{C} 2 \mathrm{~B}-\mathrm{Os} 1$ & $71.4(5)$ & $\mathrm{C} 1 \mathrm{~A}-\mathrm{Os} 1-\mathrm{C} 4 \mathrm{~A}$ & $81.7(3)$ \\
\hline $\mathrm{C} 1 \mathrm{~B}-\mathrm{C} 2 \mathrm{~B}-\mathrm{H} 2 \mathrm{~B}$ & 120 & $\mathrm{C} 5 \mathrm{~A}-\mathrm{Os} 1-\mathrm{C} 4 \mathrm{~A}$ & $37.9(2)$ \\
\hline $\mathrm{C} 3 \mathrm{~B}-\mathrm{C} 2 \mathrm{~B}-\mathrm{H} 2 \mathrm{~B}$ & 120 & $\mathrm{C} 2 \mathrm{~A}-\mathrm{Os} 1-\mathrm{C} 4 \mathrm{~A}$ & $68.4(3)$ \\
\hline $\mathrm{Os} 1-\mathrm{C} 2 \mathrm{~B}-\mathrm{H} 2 \mathrm{~B}$ & 128.6 & $\mathrm{O} 2-\mathrm{Os} 1-\mathrm{C} 3 \mathrm{~A}$ & $158.7(5)$ \\
\hline $\mathrm{C} 4 \mathrm{~B}-\mathrm{C} 3 \mathrm{~B}-\mathrm{C} 2 \mathrm{~B}$ & 120 & $\mathrm{O} 1-\mathrm{O} 1-\mathrm{C} 3 \mathrm{~A}$ & $97.2(5)$ \\
\hline $\mathrm{C} 4 \mathrm{~B}-\mathrm{C} 3 \mathrm{~B}-\mathrm{Os} 1$ & $72.4(7)$ & $\mathrm{C} 6 \mathrm{~A}-\mathrm{Os} 1-\mathrm{C} 3 \mathrm{~A}$ & $81.7(3)$ \\
\hline $\mathrm{C} 2 \mathrm{~B}-\mathrm{C} 3 \mathrm{~B}-\mathrm{Os} 1$ & $71.2(5)$ & $\mathrm{C} 1 \mathrm{~A}-\mathrm{Os} 1-\mathrm{C} 3 \mathrm{~A}$ & $69.0(3)$ \\
\hline $\mathrm{C} 4 \mathrm{~B}-\mathrm{C} 3 \mathrm{~B}-\mathrm{H} 3 \mathrm{~B}$ & 120 & $\mathrm{C} 5 \mathrm{~A}-\mathrm{Os} 1-\mathrm{C} 3 \mathrm{~A}$ & $68.4(3)$ \\
\hline $\mathrm{C} 2 \mathrm{~B}-\mathrm{C} 3 \mathrm{~B}-\mathrm{H} 3 \mathrm{~B}$ & 120 & $\mathrm{C} 2 \mathrm{~A}-\mathrm{Os} 1-\mathrm{C} 3 \mathrm{~A}$ & $37.9(2)$ \\
\hline Os $1-\mathrm{C} 3 \mathrm{~B}-\mathrm{H} 3 \mathrm{~B}$ & 128.7 & $\mathrm{C} 4 \mathrm{~A}-\mathrm{Os} 1-\mathrm{C} 3 \mathrm{~A}$ & $37.6(2)$ \\
\hline $\mathrm{C} 3 \mathrm{~B}-\mathrm{C} 4 \mathrm{~B}-\mathrm{C} 5 \mathrm{~B}$ & 120 & $\mathrm{O} 2-\mathrm{Os} 1-\mathrm{C} 2 \mathrm{~B}$ & $145.8(6)$ \\
\hline $\mathrm{C} 3 \mathrm{~B}-\mathrm{C} 4 \mathrm{~B}-\mathrm{C} 10 \mathrm{~B}$ & $118.4(16)$ & $\mathrm{O} 1-\mathrm{Os} 1-\mathrm{C} 2 \mathrm{~B}$ & $96.3(5)$ \\
\hline $\mathrm{C} 5 \mathrm{~B}-\mathrm{C} 4 \mathrm{~B}-\mathrm{C} 10 \mathrm{~B}$ & $121.6(16)$ & $\mathrm{O} 2-\mathrm{Os} 1-\mathrm{C} 3 \mathrm{~B}$ & $109.1(5)$ \\
\hline $\mathrm{C} 3 \mathrm{~B}-\mathrm{C} 4 \mathrm{~B}-\mathrm{Os} 1$ & $70.4(7)$ & $\mathrm{O} 1-\mathrm{O} 1-\mathrm{C} 3 \mathrm{~B}$ & $95.2(6)$ \\
\hline $\mathrm{C} 5 \mathrm{~B}-\mathrm{C} 4 \mathrm{~B}-\mathrm{Os} 1$ & $72.5(8)$ & $\mathrm{C} 2 \mathrm{~B}-\mathrm{Os} 1-\mathrm{C} 3 \mathrm{~B}$ & $37.4(3)$ \\
\hline $\mathrm{C} 10 \mathrm{~B}-\mathrm{C} 4 \mathrm{~B}-\mathrm{Os} 1$ & $129.3(5)$ & $\mathrm{O} 2-\mathrm{Os} 1-\mathrm{C} 1 \mathrm{~B}$ & $160.6(6)$ \\
\hline $\mathrm{C} 6 \mathrm{~B}-\mathrm{C} 5 \mathrm{~B}-\mathrm{C} 4 \mathrm{~B}$ & 120 & $\mathrm{O} 1-\mathrm{Os} 1-\mathrm{C} 1 \mathrm{~B}$ & $122.5(6)$ \\
\hline $\mathrm{C} 6 \mathrm{~B}-\mathrm{C} 5 \mathrm{~B}-\mathrm{Os} 1$ & $71.6(5)$ & $\mathrm{C} 2 \mathrm{~B}-\mathrm{Os} 1-\mathrm{C} 1 \mathrm{~B}$ & $37.3(2)$ \\
\hline $\mathrm{C} 4 \mathrm{~B}-\mathrm{C} 5 \mathrm{~B}-\mathrm{Os} 1$ & $70.7(6)$ & $\mathrm{C} 3 \mathrm{~B}-\mathrm{Os} 1-\mathrm{C} 1 \mathrm{~B}$ & $67.1(4)$ \\
\hline
\end{tabular}




$\begin{array}{ll}\text { C6B-C5B-H5B } & 120 \\ \text { C4B-C5B-H5B } & 120 \\ \text { Os1-C5B-H5B } & 130.2 \\ \text { C5B-C6B-C1B } & 120 \\ \text { C5B-C6B-Os1 } & 71.8(5) \\ \text { C1B-C6B-Os1 } & 70.6(6) \\ & \\ \text { C6A-C1A-C2A-C3A } & 0 \\ \text { C7A-C1A-C2A-C3A } & -179.8(3) \\ \text { Os1-C1A-C2A-C3A } & -53.8(5) \\ \text { C6A-C1A-C2A-Os1 } & 53.8(5) \\ \text { C7A-C1A-C2A-Os1 } & -126.0(5) \\ \text { C1A-C2A-C3A-C4A } & 0 \\ \text { Os1-C2A-C3A-C4A } & -52.4(6) \\ \text { C1A-C2A-C3A-Os1 } & 52.4(6) \\ \text { C2A-C3A-C4A-C5A } & 0 \\ \text { Os1-C3A-C4A-C5A } & -51.8(5) \\ \text { C2A-C3A-C4A-C10A } & 179.8(3) \\ \text { Os1-C3A-C4A-C10A } & 128.1(5) \\ \text { C2A-C3A-C4A-Os1 } & 51.8(5) \\ \text { C3A-C4A-C5A-C6A } & 0 \\ \text { C10A-C4A-C5A-C6A } & -179.8(3) \\ \text { Os1-C4A-C5A-C6A } & -52.5(4) \\ \text { C3A-C4A-C5A-Os1 } & 52.5(4) \\ \text { C10A-C4A-C5A-Os1 } & -127.4(5) \\ \text { C4A-C5A-C6A-C1A } & 0 \\ \text { Os1-C5A-C6A-C1A } & -53.8(5) \\ \text { C4A-C5A-C6A-Os1 } & 53.8(5) \\ \text { C2A-C1A-C6A-C5A } & 0 \\ \text { C7A-C1A-C6A-C5A } & 179.8(3) \\ \text { Os1-C1A-C6A-C5A } & 54.5(4) \\ \text { C2A-C1A-C6A-Os1 } & -54.5(4) \\ \text { C7A-C1A-C6A-Os1 } & 125.3(5) \\ \text { C2A-C1A-C7A-C9A } & -93.8(15) \\ \text { C6A-C1A-C7A-C9A } & 86.4(16) \\ \text { Os1-C1A-C7A-C9A } & 174.1(14) \\ \text { C2A-C1A-C7A-C8A } & 32.8(16) \\ \text { C6A-C1A-C7A-C8A } & -147.0(15) \\ \text { Os1-C1A-C7A-C8A } & -59(2) \\ \text { C6B-C1B-C2B-C3B } & 0 \\ \text { C7B-C1B-C2B-C3B } & 180.0(3) \\ \text { Os1-C1B-C2B-C3B } & 55.1(6) \\ \text { C6B-C1B-C2B-Os1 } & -55.1(6) \\ \text { C7B-C1B-C2B-Os1 } & 124.8(6) \\ \text { C1B-C2B-C3B-C4B } & 0 \\ \text { Os1-C2B-C3B-C4B } & 55.5(8) \\ \text { C1B-C2B-C3B-Os1 } & -55.5(8)\end{array}$

$\begin{array}{ll}\text { O2-Os1-C4B } & 87.4(4) \\ \text { O1-Os1-C4B } & 119.8(6) \\ \text { C2B-Os1-C4B } & 67.1(4) \\ \text { C3B-Os1-C4B } & 37.2(3) \\ \text { C1B-Os1-C4B } & 78.8(4)\end{array}$

$\mathrm{C} 2 \mathrm{~B}-\mathrm{C} 3 \mathrm{~B}-\mathrm{C} 4 \mathrm{~B}-\mathrm{C} 5 \mathrm{~B} \quad 0$

$\mathrm{Os} 1-\mathrm{C} 3 \mathrm{~B}-\mathrm{C} 4 \mathrm{~B}-\mathrm{C} 5 \mathrm{~B} \quad 55.0$ (5)

$\mathrm{C} 2 \mathrm{~B}-\mathrm{C} 3 \mathrm{~B}-\mathrm{C} 4 \mathrm{~B}-\mathrm{C} 10 \mathrm{~B} \quad-179.9(3)$

Os1-C3B-C4B-C10B $\quad-124.9(6)$

$\mathrm{C} 2 \mathrm{~B}-\mathrm{C} 3 \mathrm{~B}-\mathrm{C} 4 \mathrm{~B}-\mathrm{Os} 1 \quad-55.0(5)$

$\mathrm{C} 3 \mathrm{~B}-\mathrm{C} 4 \mathrm{~B}-\mathrm{C} 5 \mathrm{~B}-\mathrm{C} 6 \mathrm{~B} \quad 0$

$\mathrm{C} 10 \mathrm{~B}-\mathrm{C} 4 \mathrm{~B}-\mathrm{C} 5 \mathrm{~B}-\mathrm{C} 6 \mathrm{~B} \quad 179.8$ (3)

Os1-C4B-C5B-C6B 54.0 (5)

$\mathrm{C} 3 \mathrm{~B}-\mathrm{C} 4 \mathrm{~B}-\mathrm{C} 5 \mathrm{~B}-\mathrm{Os} 1 \quad-54.0$ (5)

$\mathrm{C} 10 \mathrm{~B}-\mathrm{C} 4 \mathrm{~B}-\mathrm{C} 5 \mathrm{~B}-\mathrm{Os} 1 \quad 125.9$ (5)

$\mathrm{C} 4 \mathrm{~B}-\mathrm{C} 5 \mathrm{~B}-\mathrm{C} 6 \mathrm{~B}-\mathrm{C} 1 \mathrm{~B} \quad 0$

Os1-C5B-C6B-C1B $53.6(7)$

$\mathrm{C} 4 \mathrm{~B}-\mathrm{C} 5 \mathrm{~B}-\mathrm{C} 6 \mathrm{~B}-\mathrm{Os} 1 \quad-53.6$ (7)

$\mathrm{C} 2 \mathrm{~B}-\mathrm{C} 1 \mathrm{~B}-\mathrm{C} 6 \mathrm{~B}-\mathrm{C} 5 \mathrm{~B} \quad 0$

$\mathrm{C} 7 \mathrm{~B}-\mathrm{C} 1 \mathrm{~B}-\mathrm{C} 6 \mathrm{~B}-\mathrm{C} 5 \mathrm{~B} \quad-180.0(3)$

Os1- C1B-C6B-C5B $\quad-54.2(5)$

$\mathrm{C} 2 \mathrm{~B}-\mathrm{C} 1 \mathrm{~B}-\mathrm{C} 6 \mathrm{~B}-\mathrm{Os} 1 \quad 54.2(5)$

$\mathrm{C} 7 \mathrm{~B}-\mathrm{C} 1 \mathrm{~B}-\mathrm{C} 6 \mathrm{~B}-\mathrm{Os} 1 \quad-125.8(6)$

$\mathrm{C} 2 \mathrm{~B}-\mathrm{C} 1 \mathrm{~B}-\mathrm{C} 7 \mathrm{~B}-\mathrm{C} 9 \mathrm{~B} \quad 93.9(19)$

$\mathrm{C} 6 \mathrm{~B}-\mathrm{C} 1 \mathrm{~B}-\mathrm{C} 7 \mathrm{~B}-\mathrm{C} 9 \mathrm{~B} \quad-86.1(19)$

Os1-C1B-C7B-C9B $\quad-176.3(18)$

$\mathrm{C} 2 \mathrm{~B}-\mathrm{C} 1 \mathrm{~B}-\mathrm{C} 7 \mathrm{~B}-\mathrm{C} 8 \mathrm{~B} \quad-32(2)$

$\mathrm{C} 6 \mathrm{~B}-\mathrm{C} 1 \mathrm{~B}-\mathrm{C} 7 \mathrm{~B}-\mathrm{C} 8 \mathrm{~B} \quad 148$ (2)

Os1-C1B-C7B-C8B 58 (3)

$\mathrm{O} 1-\mathrm{C} 11-\mathrm{C} 12-\mathrm{C} 13 \quad-178.9(11)$

$\mathrm{C} 17-\mathrm{C} 11-\mathrm{C} 12-\mathrm{C} 13 \quad 1.2(18)$

$\mathrm{C} 11-\mathrm{C} 12-\mathrm{C} 13-\mathrm{C} 14$

$\mathrm{C} 12-\mathrm{C} 13-\mathrm{C} 14-\mathrm{C} 15 \quad-1(2)$

$\mathrm{C} 13-\mathrm{C} 14-\mathrm{C} 15-\mathrm{C} 16 \quad-2(2)$

$\mathrm{C} 14-\mathrm{C} 15-\mathrm{C} 16-\mathrm{C} 17 \quad 0$ (2)

$\mathrm{C} 15-\mathrm{C} 16-\mathrm{C} 17-\mathrm{O} 2 \quad-176.9(11)$

$\mathrm{C} 15-\mathrm{C} 16-\mathrm{C} 17-\mathrm{C} 11 \quad 4.9(18)$

$\mathrm{O} 1-\mathrm{C} 11-\mathrm{C} 17-\mathrm{O} 2 \quad-4.0(12)$

$\mathrm{C} 12-\mathrm{C} 11-\mathrm{C} 17-\mathrm{O} 2 \quad 176.0(9)$

$\mathrm{O} 1-\mathrm{C} 11-\mathrm{C} 17-\mathrm{C} 16 \quad 174.3(9)$

$\mathrm{C} 12-\mathrm{C} 11-\mathrm{C} 17-\mathrm{C} 16 \quad-5.8(16)$

$\mathrm{C} 12-\mathrm{C} 11-\mathrm{O} 1-\mathrm{Os} 1 \quad-176.6(7)$

$\mathrm{C} 17-\mathrm{C} 11-\mathrm{O} 1-\mathrm{Os} 1 \quad 3.4(10)$

$\mathrm{C} 16-\mathrm{C} 17-\mathrm{O} 2-\mathrm{Os} 1 \quad-175.7$ (7)

$\mathrm{C} 11-\mathrm{C} 17-\mathrm{O} 2-\mathrm{Os} 1 \quad 2.6(10)$ 
supporting information

Hydrogen-bond geometry $\left(A,{ }^{\circ}\right)$

\begin{tabular}{lllll}
\hline$D-\mathrm{H} \cdots A$ & $D-\mathrm{H}$ & $\mathrm{H} \cdots A$ & $D \cdots A$ & $D-\mathrm{H} \cdots A$ \\
\hline $\mathrm{C} 2 A-\mathrm{H} 2 A \cdots \mathrm{O} 1^{\mathrm{i}}$ & 0.95 & 2.40 & $3.24(2)$ & 148 \\
$\mathrm{C} 3 B-\mathrm{H} 3 B \cdots \mathrm{O} 1^{\mathrm{i}}$ & 0.95 & 2.75 & $3.38(2)$ & 124 \\
$\mathrm{C} 5 B-\mathrm{H} 5 B \cdots \mathrm{O} 2^{\mathrm{ii}}$ & 0.95 & 2.50 & $3.25(2)$ & 136 \\
$\mathrm{C} 6 A-\mathrm{H} 6 A \cdots \mathrm{O} 2^{\mathrm{ii}}$ & 0.95 & 2.71 & $3.39(2)$ & 124 \\
$\mathrm{C} 5 A-\mathrm{H} 5 A \cdots \mathrm{O} 2^{\mathrm{ii}}$ & 0.95 & 2.77 & $3.39(2)$ & 124
\end{tabular}

Symmetry codes: (i) $-x,-y+2,-z$; (ii) $-x+1,-y+2,-z$. 